\title{
Deformed Hartree-Fock Calculation of Proton-Rich Nuclei*
}

\author{
N. Tajima, N. Onishi, and S. Takahara \\ Institute of Physics, College of Arts and Sciences, \\ University of Tokyo, Komaba, Meguro-ku, Tokyo, 153, Japan
}

November 1, 1994

\begin{abstract}
We perform Hartree-Fock+BCS calculations for even-even nuclei with $2 \leq Z \leq 82$ and $N$ ranging from outside the proton drip line to the experimental frontier on the neutron-rich side. The ground state solutions are obtained for 737 nuclei, together with shape-coexistence solutions for 480 nuclei. Our method features the Cartesian-mesh representation of single-particle wavefunctions, which is advantageous in treating nucleon skins and exotic shapes. The results are compared with those of the finite-range droplet model of Møller et al. as well as the experimental values.
\end{abstract}

\section{INTRODUCTION}

Several theoretical frameworks have been presented for the sake of global (on the $N-Z$ plane) calculations of nuclear ground state properties. One of the most elaborate results is given by Møller et al. [1] using the finiterange droplet model with a microscopic shell correction (FRDM). Another

*To be published in a special issue of Nuclear Physics $\mathbf{A}$ for the proceedings of the international symposium on physics of unstable nuclei (Niigata, Japan, October 31 - November 3, 1994) edited by H. Horiuchi, K. Ikeda, K. Sato, Y. Suzuki, and I. Tanihata 
extensive calculation is carried out by Aboussir et al. [2] in the extended Thomas-Fermi approach. The former as well as the latter methods can be regarded as approximations to the Hartree-Fock (HF) equation. While meanfield calculations under spherical symmetry are very easily done nowadays, those including deformation still requires long computation time for global calculations.

In this paper we show the results of our global HF+BCS calculations and compare them with those of FRDM and experiment. Most of the methods to treat deformed nuclei[1, 2, 3] express single-particle wavefunctions by expansion in a harmonic oscillator basis. In contrast, we use a $\mathrm{HF}+\mathrm{BCS}$ code ev8[4], which features a Cartesian-mesh representation of single-particle wavefunctions. The mesh representation is expected to be suitable for describing the atomic nucleus because the saturation property of nuclear matter guarantees the suppression of large-momentum components in wavefunctions.

The advantage of the mesh representation for the study of unstable nuclei is the capability to treat nucleon skins and halos, while they cannot be described correctly in the oscillator basis expansion since the asymptotic form of wavefunctions far from the nuclear surface is determined by the basis.

Another advantage of the mesh representation is that it can treat exotic (e.g. high-multipole) shapes and large (e.g. super and hyper) deformations without preparing a special basis for each shape.

\section{METHOD OF CALCULATION}

In the code $e v 8$, an octant of a nucleus is placed in a corner of a box $(13 \times$ $13 \times 14 \mathrm{fm}^{3}$ ), imposing a symmetry with respect to reflections in $x-y, y-z$, and $z$-x planes (the point group $D_{2 h}$ ). The mesh size takes on $1 \mathrm{fm}$. The correction for the inaccuracy due to this finite mesh size is necessary only for the total binding energy 4 . (We need a precision of $0.1 \mathrm{MeV}$ in the total kinetic energy of $4 \mathrm{GeV}$ for ${ }^{208} \mathrm{~Pb}$.) The correction to be added is,

$$
\Delta E(Z, N)=0.00595 \cdot(Z+N)+0.1090 \cdot(N-Z)+1.24 \quad[\mathrm{MeV}],
$$

which was obtained by fitting to the outputs of a spherical HF+BCS code [5 with sufficiently small grid size $(0.2 \mathrm{fm})$. In Fig. 1, the nucleon density of a nucleus ${ }^{138}$ Dy in the $x-z$ plane is shown as an illustration of our mesh representation. 
The code uses the Skyrme interaction. Among the widely used parameters of the interaction, we choose the SIII[6]. Its validity has been examined in many nuclear structure calculations. In particular, its single-particle spectrum is in good agreement with experiment. It also reproduces fairly well the $N-Z$ dependence of the binding energy compared with other widely-used parameter sets[7]. On the other hand, its incompressibility is said to be too large.

The pairing correlation plays an important role in determining the nucleon drip lines 8]. It also influences the deformation, sometimes strongly. For example, one can see in ref. [9] that the potential energy curve of ${ }^{186} \mathrm{~Pb}$ is substantially changed when the pairing interaction strength is slightly reduced. Therefore, it should be kept in mind that more accurate treatment of the pairing is necessary for deformed and/or near-drip-line nuclei than for spherical stable nuclei.

The BCS method cannot be applied to nuclei near the neutron drip line because the Fermi energy is so high that positive energy HF orbitals, whose wavefunctions are extended over the box, are occupied through pairscattering processes. This brings about an unphysical situation in which neutron gas surrounds the nucleus. This problem is resolved in the HartreeFock-Bogolyubov (HFB) theory, in which ordinary and pair densities are localized when the Fermi level is negative 8].

On the other hand, for nuclei near the proton drip line, the $\mathrm{HF}+\mathrm{BCS}$ method is still useful to obtain localized solutions because the Coulomb barrier keeps the wavefunctions of positive energy HF orbitals localized spatially. In practice, to prevent the tunneling through the barrier, we modify the Coulomb potential outside the barrier so that it is higher than the cut-off energy $\left(\lambda_{\mathrm{HF}}^{\tau}+7.3 \mathrm{MeV}\right)$ of the pairing interaction.

We use a seniority pairing force, whose pair-scattering matrix elements are defined as a constant multiplied by cut-off factors depending on the singleparticle energy $\epsilon_{i}$ :

$$
\begin{aligned}
\left\langle i \bar{\imath}\left|V_{\mathrm{pair}}^{\tau}\right| j \bar{\jmath}\right\rangle & =-G_{\tau} f_{\tau}\left(\epsilon_{i}\right) f_{\tau}\left(\epsilon_{j}\right), \\
f_{\tau}(\epsilon) & =\left\{1+\exp \frac{\epsilon-\lambda_{\mathrm{HF}}^{\tau}-5 \mathrm{MeV}}{0.5 \mathrm{MeV}}\right\}^{-1 / 2} \theta\left(\lambda_{\mathrm{HF}}^{\tau}+7.3 \mathrm{MeV}-\epsilon\right),(3)
\end{aligned}
$$

where $\tau$ means proton or neutron while $\lambda_{\mathrm{HF}}^{\tau}$ is the Fermi level of the HF (normal) state. For neutrons, the righthand side of eq. (3) is multiplied 
furthermore by $\theta(-\epsilon)$. We need a prescription to determine the strength $G_{\tau}$ for each nucleus in the entire region of the nuclear chart. For this purpose, we develop a method based on the continuous spectrum approximation using the Thomas-Fermi single-particle level density. As the average pairing gap, we adopt the classical empirical formula $\Delta=12 \mathrm{MeV} / \sqrt{A}$.

The imaginary-time time-dependent-Hartree-Fock evolution method 10 is used to obtain the solutions to the HF+BCS equation. Usually, 500-2000 steps are necessary to obtain a solution for each nucleus, where the time step is chosen as $1.5 \times 10^{-24} \mathrm{sec}$.

In order to search a prolate (an oblate) solution, we first exert an external potential proportional to $Q_{\mathrm{z}}$ on the initial wavefunction until its quadrupole deformation parameter,

$$
\delta_{2}=3\left\langle Q_{\mathrm{z}}\right\rangle / 4\left\langle r^{2}\right\rangle,
$$

satisfies $\delta_{2}>0.1\left(\delta_{2}<-0.1\right)$. Then, we switch off the external potential, let the wavefunction evolve by itself, and see if it converges to a deformed local minimum.

\section{RESULTS}

We have performed the HF+BCS calculations with the Skyrme SIII force for 752 even-even nuclei with $2 \leq Z \leq 82$ and, for each $Z, N$ ranging from several units less than the proton drip line to the heaviest isotope observed. The extension of the calculation to the neutron drip line will be deferred until a deformed HFB method is developed. Spatially localized solutions are obtained for 737 nuclei. Note that some of them are outside the proton drip line: The localization is due to the lifting of the Coulomb potential outside the barrier. We have also searched for prolate and oblate shape-coexistence solutions and found the second minimum for 480 nuclei.

Although our method allows triaxial shapes, all the solutions we obtained are axially symmetric: The $Y_{2,2}$ as well as the $Y_{4,2}$ deformations are almost vanishing, while the $Y_{4,4}$ deformation is larger but very small. It is possible, however, that local minima exist at $\gamma \neq 0^{\circ}$ points in the $\beta-\gamma$ plane which are separated from the axial path by a potential barrier.

Among the quantities which can be extracted from our results are the binding energy, even-multipole deformation parameters, radius, skin thick- 
ness (halo is not found), single-particle levels, and pairing gaps. We also have the information on the prolate-oblate difference of these quantities.

In Fig. 2, the quadrupole deformation parameter $\left|\delta_{2}\right|$ is shown for the ground states of nuclei with $42 \leq Z \leq 58$. One can see the development of deformation with the increase of the number of valence particles and holes. One can also see that oblate minima are competing with prolate ones for not very large deformations $\left(\left|\delta_{2}\right|<0.2\right)$. By comparing Fig. 2 with the result of FRDM[目], we find conspicuous differences for $40<Z, N<50$, where only the latter predicts very large deformations.

In Fig. 3, two-proton separation energies are plotted for nuclei in the neighbourhood of ${ }^{100} \mathrm{Sn}$. The results of FRDM 11 and experimental values 11 are also shown. The agreement between them looks more impressive considering that the SIII force was made in 1975 while FRDM was revised in 1993. A noticeable discrepancy is that the jump across the $Z=50$ major shell closure is smaller in experiment than our calculation.

In Fig. 田, the thickness of the proton skin is shown. The definition of the skin is taken from ref. [12]: The condition for a point $\boldsymbol{r}$ to be in the proton skin is,

$$
\rho_{\mathrm{p}}(\boldsymbol{r})>4 \rho_{\mathrm{n}}(\boldsymbol{r}) \quad \text { and } \quad \rho_{\mathrm{p}}(\boldsymbol{r})>\rho_{\mathrm{p}}(0) / 100 .
$$

When the nucleus is deformed, the thickness of the skin can depend on the direction. In the figure the skin thickness in the $z$-axis (symmetry axis) is plotted. The skin in the $x-y$ (equatorial) plane is usually slightly thinner for prolate shapes because of the Coulomb repulsion. One can see that the proton skin is very thin $(<0.3 \mathrm{fm})$ for nuclei inside the proton drip line except some light $(Z \leq 20)$ nuclei. For all the nuclei with $50<Z \leq 82$, which are not shown in the figure, the proton skin does not exist.

We thank Dr. P. Bonche, Dr. H. Flocard, and Dr. P.-H. Heenen for providing the Hartree-Fock+BCS code ev8 on a Cartesian mesh. We are grateful to Dr. Flocard for useful discussions, too. The computation was financially supported by RCNP, Osaka University, as RCNP Computational Nuclear Physics Project (No. 94-B-01). 


\section{References}

[1] P. Møller, J.R. Nix, W.D. Myers and W.J. Swiatecki, Atomic Data Nucl. Data Tables (1994), in press.

[2] Y. Aboussir, J.M. Pearson, A.K. Dutta and F. Tondeur, Nucl. Phys. A549 (1992) 155.

[3] J. Dechargé and D. Gogny, Phys. Rev. C21 (1980) 1568.

[4] P. Bonche, H. Flocard, P.-H. Heenen, S.J. Krieger and M.S. Weiss, Nucl.Phys. A443 (1985) 39.

[5] P.-G. Reinhard, in "Computational Nuclear Physics 1", ed. K. Langanke, J.A. Maruhn, and S.E. Koonin, Springer-Verlag (Berlin) 1991.

[6] M. Beiner, H. Flocard, Nguyen van Giai and P. Quentin, Nucl.Phys. A238 (1975) 29.

[7] N. Tajima, P. Bonche, H. Flocard, P.-H. Heenen, and M.S. Weiss, Nucl. Phys. A551 (1993) 434.

[8] J. Dobaczewski, H. Flocard, and J. Treiner, Nucl.Phys. A422 (1984) 103.

[9] N. Tajima, H. Flocard, P. Bonche J. Dobaczewski, and P.-H. Heenen, Nucl. Phys. A551 (1993) 409.

[10] K.T.R. Davies, H. Flocard, S. Krieger, and M.S. Weiss, Nucl. Phys. A342 (1980) 111.

[11] G. Audi, Midstream atomic mass evaluation (1989), in ref. [1].

[12] N. Fukunishi, T. Otsuka, and I. Tanihata, Phys. Rev. C48 (1993) 1648. 


\section{FIGURE CAPTIONS}

Fig. 1. The nucleon density of ${ }^{138} \mathrm{Dy}$ in the $x-z$ plane calculated with the $\mathrm{HF}+\mathrm{BCS}$ method with the SIII force. This nucleus is located just inside the proton drip line and has a prolate shape $\left(\delta_{2}=0.315\right)$ which is symmetric in the $z$-axis. Dashed lines correspond to the $1 \mathrm{fm}$ mesh.

Fig. 2. The quadrupole deformation parameter $\left|\delta_{2}\right|$ as a function of the neutron number $N$ for some isotope chains near Sn calculated with the $\mathrm{HF}+\mathrm{BCS}$ method with the SIII force. On the lefthand (righthand) side, $Z=42,44,46,48(Z=58,56,54,52)$ isotopes are designated by triangles connected with solid lines, squares with short-dash lines, diamond shapes with long-dash lines, and circles with dot-dash lines, respectively. The solid symbols are used for prolate states, while open symbols are used for oblate ones. All the $Z=50$ isotopes are spherical and have no deformed local minima.

Fig. 3. Two-proton separation energy in the neighborhood of ${ }^{100} \mathrm{Sn}$. The calculation with the HF+BCS method with the SIII force is designated by solid circles. The solid lines connect the solid circles of the same neutron number. The neutron number is printed at an end of each line. The results of FRDM [1] are represented by open circles, while the experimental values [11] are designated by plus marks.

Fig. 4. The thickness of the proton skin in the z-axis (the symmetry axis) calculated with the HF+BCS method with the SIII force. The definition of the skin is given in the text. The solid (open) circles are used for nuclei with $S_{2 \mathrm{p}}>0\left(S_{2 \mathrm{p}}<0\right)$. The lines connect isotones. The number printed near each line means the neutron number of the isotone chain. 


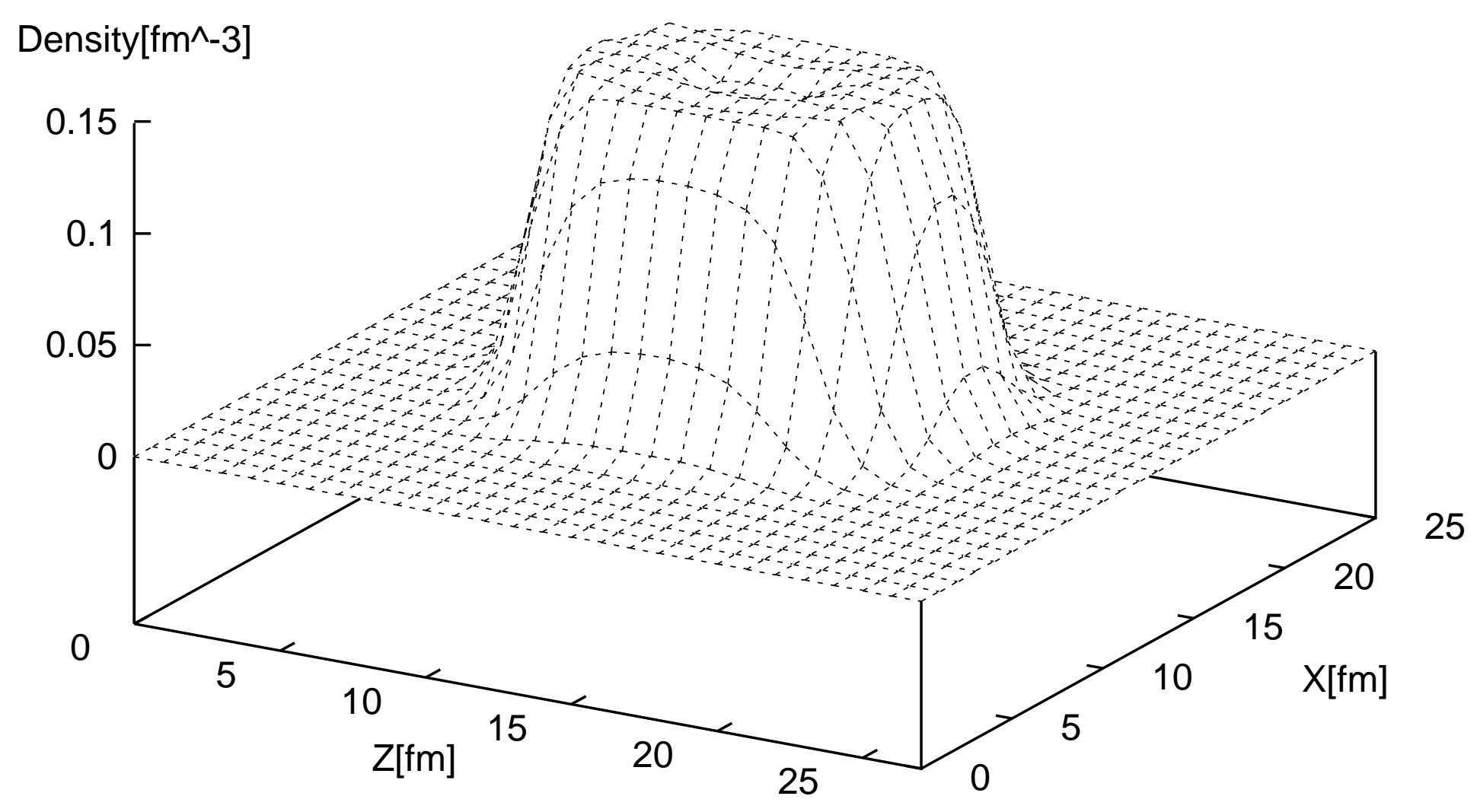




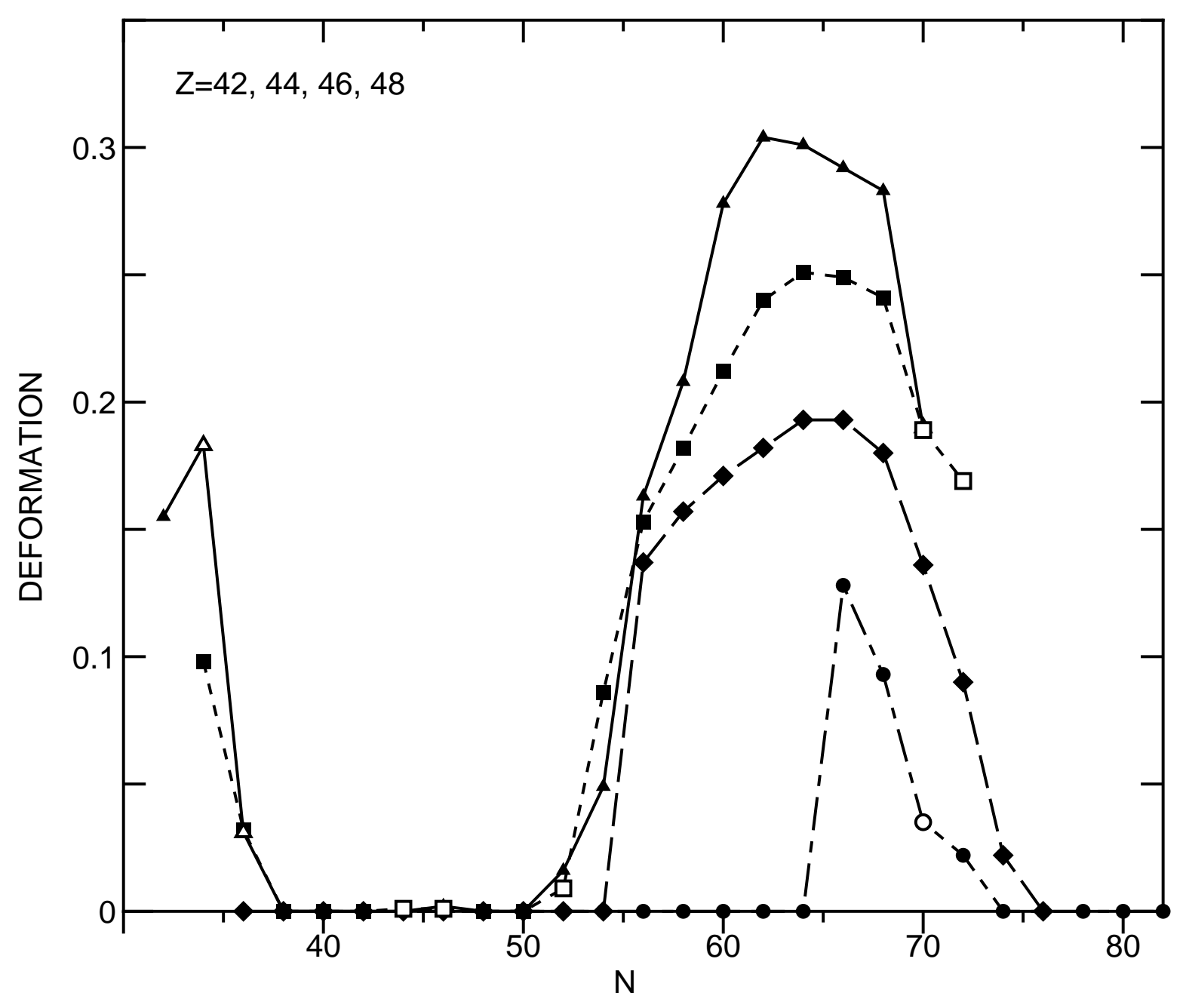




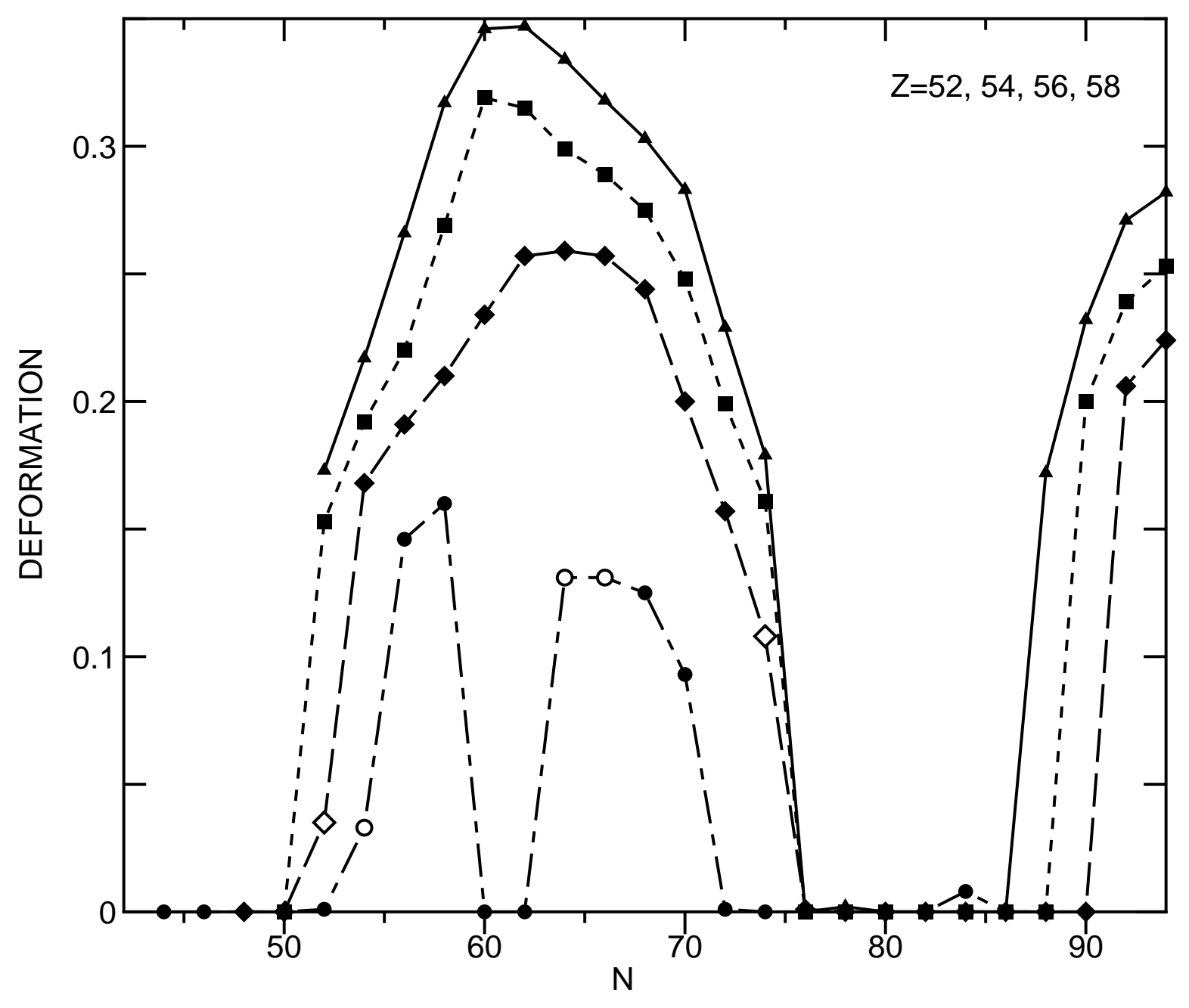




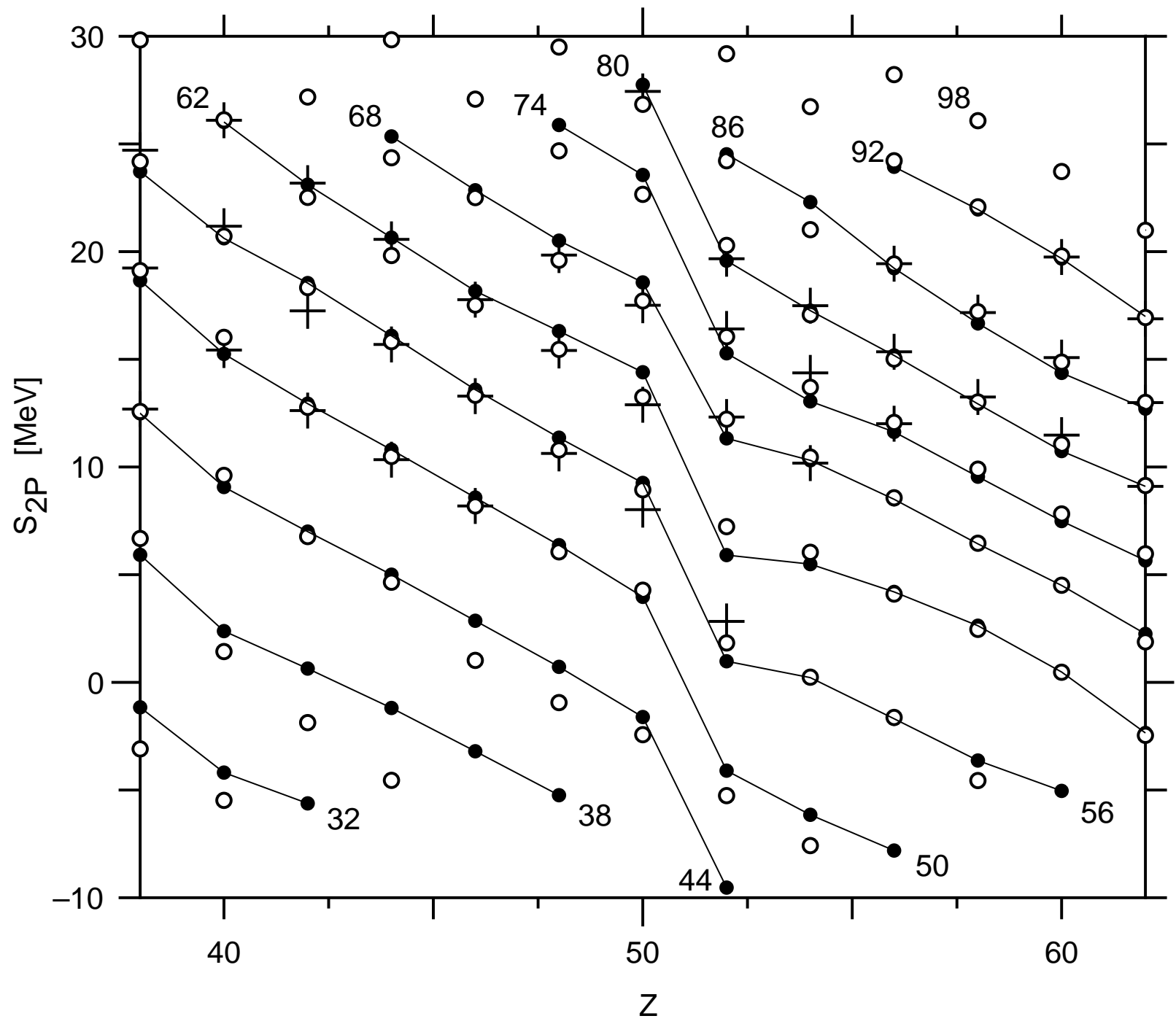




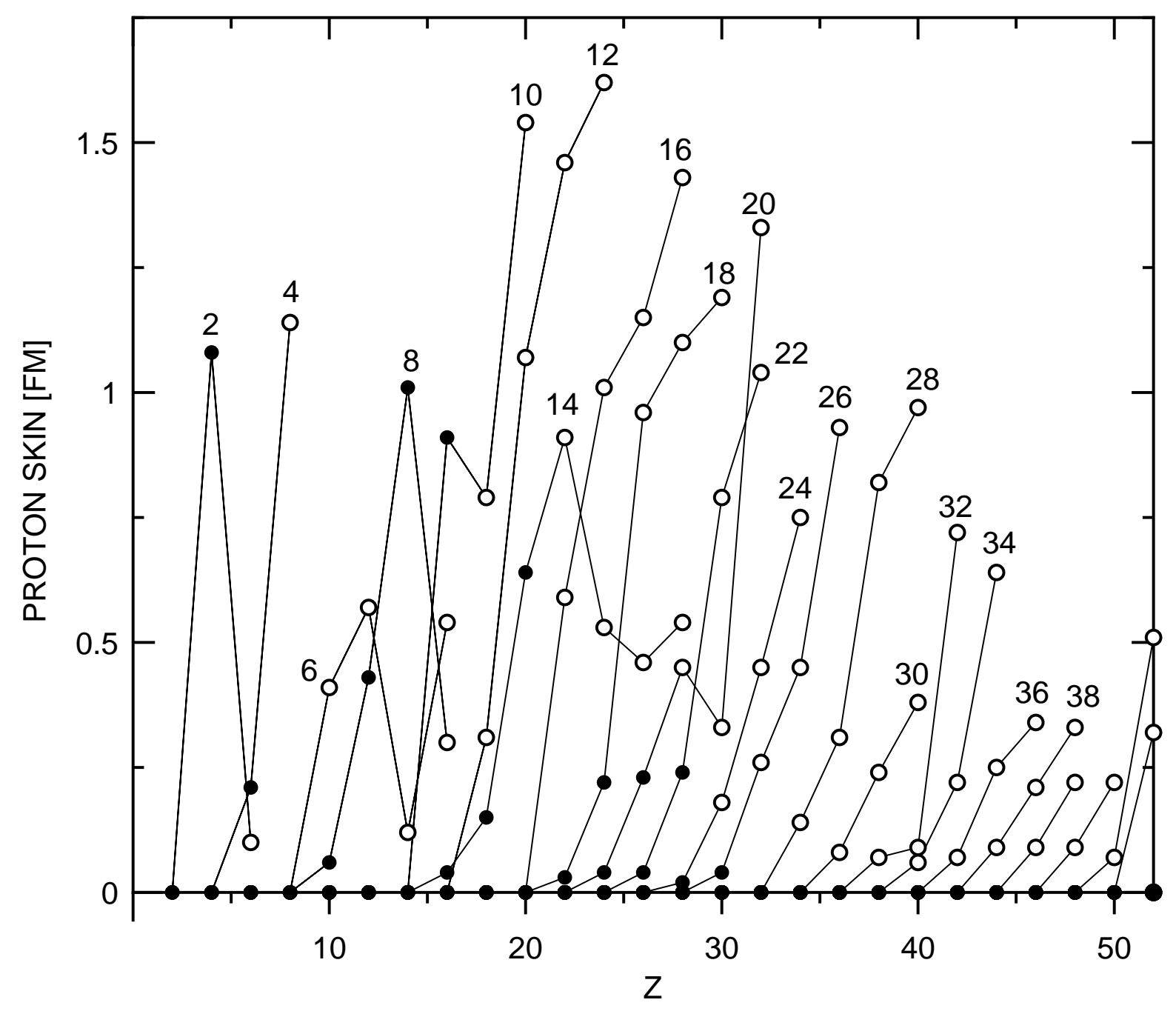

